\title{
The impact of head gradient transients on transport in heterogeneous formations: Application to the Borden Site
}

\author{
Alberto Bellin \\ Dipartimento di Ingegneria Civile ed Ambientale, Università di Trento, Trento, Italy \\ Gedeon Dagan \\ Faculty of Engineering, Tel Aviv University, Tel Aviv, Israel \\ Yoram Rubin \\ Department of Civil and Environmental Engineering, University of California at Berkeley
}

\begin{abstract}
A three-dimensional interpretation of the Borden Site experiment is proposed with the aid of a recently developed stochastic model that incorporates transiency of the piezometric head gradient. The behavior of the second-order central transverse plume moments is analyzed with the aim of explaining the underprediction of experimental results by existing steady state models. The model assumes uniformity in space, but time varying mean head gradient, stationary and anisotropic log conductivity, and a first-order approximation in the log conductivity variance. The solution for the trajectory covariances, assumed to be equal to the plume spatial second moments under ergodic conditions, is evaluated with the aid of a few quadratures. An analysis of the parameters and plume spatial moments found in the literature precedes application of the model. It is found that unsteadiness leads to an increase in the transverse, horizontal, second moment compared with the one based on a steady state flow model. Still, application of Borden Site data leads to values lower than the ones inferred from concentration measurements. We conclude that unsteadiness of the mean head gradient does not fully explain the magnitude of observed transverse spreading. However, the impact of transients on spreading is significant in the transverse direction, and the definition of a Fickian transverse dispersion coefficient may not be a simple task for transport occurring under natural flow conditions.
\end{abstract}

\section{Background}

The Borden Site field test [Sudicky, 1986; Freyberg, 1986; Mackay et al., 1986] is one of the first large-scale transport experiments in which measurements of solute concentration and aquifer properties were carried out in a detailed manner. One of the main aims of this experiment was to validate existing theories that relate the solute spreading pattern to spatial variability of medium properties (mainly the hydraulic conductivity $K$ ). Limiting the discussion to conservative solutes, the procedure was (1) to derive the first and second spatial moments of the solute plume as functions of time by using the measured solute concentrations; (2) to determine independently the statistical parameters of $Y=\ln K$, regarded as a normal random space function; (3) to derive the head gradient from head measurements; and (4) to compare the theoretical spatial moments which are based on the above with the ones inferred from measurements.

In the first attempts along these lines [Freyberg, 1986; Sudicky, 1986], the concentration values employed in calculations were related to the discrete spatial measurements by an interpolation scheme (see section 3 ). The parameters characterizing $Y$, namely, the geometric mean $K_{G}$, the variance $\sigma_{Y}^{2}$, and Copyright 1996 by the American Geophysical Union.

Paper number 96WR01629.

0043-1397/96/96WR-01629\$09.00 the horizontal and vertical integral scales $I_{Y h}$ and $I_{Y v}$, were in turn determined by fitting an exponential covariance function $C_{Y}$ to the data (see Table 1). The theoretical values of the spatial moments of the plume were evaluated by using the first-order Lagrangian approximation for particle trajectories [Dagan, 1982; Dagan, 1984] for steady two-dimensional flow and transport, after multiplying the variance $\sigma_{Y}^{2}$ by a factor of 0.74 , based on the conjecture that thin horizontal layers of low hydraulic conductivity were preventing vertical flow and the assumed mean distance between layers was $I_{Y v}$. The conjecture was supported indirectly by the observed very small vertical spread of the plume and by the good agreement between inferred and computed horizontal second spatial moments, $S_{11}$ (in the direction of the mean flow) and $S_{22}$ (transverse). In particular, the asymptotic logarithmic growth of the theoretical $X_{22}$ with travel time, as compared with its tendency to a constant value for the three-dimensional (3-D) solution, led to good agreement with measurements.

Subsequent attempts have been made to improve the identification of hydraulic properties, the spatial moments computation from concentration measurement, and the theoretical tools. Recently, the statistical parameters of $Y$, as well as their estimation variances, were derived by Woodbury and Sudicky [1991] based on the two vertical transects $\mathrm{AA}^{\prime}$ and $\mathrm{BB}^{\prime}$ along which $K$ was measured (see Table 1 ). These estimates were found to differ from the initial aforementioned estimates. Similarly, improved estimates and errors of estimation for the 
Table 1. Log Conductivity Parameters

\begin{tabular}{llllll}
\hline Parameter & A-A $^{\prime}$ & s.d. & B-B' $^{\prime}$ & s.d. & SUD \\
\hline$\hat{\sigma}_{Y}^{2}$ & 0.244 & 0.008 & 0.366 & 0.019 & 0.38 \\
$\sigma_{0}^{2}$ & 0.072 & 0.021 & 0.111 & 0.16 & 0.10 \\
$I_{Y h}, \mathrm{~m}$ & 5.14 & 1.17 & 8.33 & 1.68 & 2.8 \\
$I_{Y v}, \mathrm{~m}$ & 0.209 & 0.050 & 0.336 & 0.075 & 0.12 \\
\hline
\end{tabular}

Here $\hat{\sigma}_{Y}^{2}$ is the total $\log$ conductivity variance, $\sigma_{0}^{2}$ is the variance related to the "nugget" effect, and $I_{Y h}$ and $I_{Y v}$ are the horizontal and vertical integral scales, respectively. SUD refers to values obtained by Sudicky [1986], and s.d. is the standard deviation. Woodbury and Sudicky [1991] estimated $K_{G}$ and $n$ at $7.17 \mathrm{~cm} / \mathrm{s}$ and 0.33 , respectively. After Woodbury and Sudicky [1991].

plume spatial moments were provided by Barry and Sposito [1990], Rajaram and Gelhar [1991], and Thierrin and Kitanidis [1994] (for details, see section 3). For $S_{11}$ these estimates were quite similar, but significant scatter was observed for the transverse $S_{22}$ (see Figure 6). Finally, since there was no direct evidence to support the conjecture of two-dimensional (2-D) transport, theoretical 3-D solutions of flow and transport for anisotropic $C_{Y}$ [e.g., Dagan, 1988] were also tested [Woodbuny and Sudicky, 1991]. The picture that emerged from these efforts with regard to longitudinal spreading was that the theoretical $X_{11}$ overestimates the inferred $S_{11}$ by as much as a factor of 2 , due to the increase in the new estimate of $I_{Y h}$ (Table 1). This effect was recently [Burr et al., 1994; Dagan, 1995; Fiori, 1996] attributed to the influence of transverse pore-scale dispersion in the vertical direction, which was neglected previously. Examination of Figures 1 and 2 of Fiori [1996] shows that for an anisotropy ratio $f=I_{Y v} / I_{Y h}=0.05$, which is close to the one prevailing at the Borden Site (Table 1 ), the longitudinal second moment $X_{11}$ is reduced by a factor of 0.6 compared with purely advective transport for $\mathrm{Pe}=$ $I_{Y v} / \alpha_{d T} \cong 250$, where $\alpha_{d T}$ is the transverse pore-scale dispersivity. With $I_{Y v} \cong 0.21 \mathrm{~m}$, the inferred value is $\alpha_{d T} \cong 0.8$ $\mathrm{mm}$. Unfortunately, the transverse pore-scale dispersivity is not known from measurements and this hypothesis could not be tested independently, but the above value of $\alpha_{d T}$ is plausible. In view of these findings and of the errors of estimation of parameters, the agreement between theory and experiment can be viewed as acceptable. The situation is different with respect to the horizontal transverse moment $S_{22}$ inferred from concentration measurements (Figure 6), which was found to grow with time and to be much larger than the theoretical $X_{22}$, derived with the aid of 3-D models. The large discrepancy between measurements and theory has been attributed recently [Sudicky, 1986; Rehfeldt and Gelhar, 1992; Naff et al., 1988, 1989; Farrell et al., 1994] to the time variations of the head gradient, resulting in transverse motion of the plume that was neglected previously. In the original Borden Site experiment (BS1), carried out in the years 1982-1985, the monitoring of heads was not detailed enough for assessing the time variations. Heads were measured on a time continuous basis later on at the same site during the period 1989-1991 (BS2), without carrying out a transport experiment. In a recent article [Farrell et al., 1994] the BS2 time record has been used in order to predict the spatial moments of a plume similar to that of BS1 by a few methods, leading to different results. In particular, enhanced transverse spreading close to the measured one in BS1 was obtained by applying the method of Rehfeldt and Gelhar [1992].

In a recent study by Dagan et al. [1995] (hereinafter referred to as DBR), the impact of unsteadiness on the head gradient on transport was investigated using a Lagrangian framework and under assumptions that differed radically from those of previous works. We also found that for 2-D and 3-D formations of isotropic heterogeneity, the unsteady component has a large impact upon the $X_{22}$. The aim of the present article is to apply DBR to the Borden Site experiments in order to assess the influence of time variations upon plume transverse spatial moment.

The plan of the article is as follows: In the next section we describe briefly, for the sake of completeness, the DBR model; subsequently, we discuss the field data we used, and finally, we derive the spatial moments under the conditions prevailing at BS1, but with time variations compatible with both BS1 and BS2.

\section{The DBR Model}

For the sake of completeness we recapitulate the assumptions and the relationships pertinent to the DBR model.

1. The $\log$ conductivity $Y(x)=\ln K(x)$ is modeled as a stationary random space function of mean $\langle Y\rangle$ and anisotropic exponential covariance function

$$
\begin{aligned}
C_{Y}(r)= & \sigma_{Y}^{2} \exp \left[-\sqrt{\left(r_{1} / I_{1}\right)^{2}+\left(r_{2} / I_{2}\right)^{2}+\left(r_{3} / I_{3}\right)^{2}}\right] \\
& +\sigma_{0}^{2}\left[1-H_{v}(r)\right]
\end{aligned}
$$

where $r_{i}, i=1, \cdots, 3$ are the components of the two points lag distance, $r=\sqrt{r_{1}^{2}+r_{2}^{2}+r_{3}^{2}}$, and $I_{1}, I_{2}, I_{3}$ are the integral scales along the three principal direction of the log conductivity covariance tensor. Here $\sigma_{0}^{2}$ is the variance related to the "nugget" effect, and $H_{v}(r)$ is the Heaviside step function.

2. The piezometric head $H$ comprises the mean $\langle H\rangle=$ $-\mathrm{J}(t) \cdot \mathbf{x}$ and the fluctuation $h(\mathbf{x}, t)$ such that $H(\mathbf{x}, t)=$ $-\mathrm{J}(t) \cdot \mathrm{x}+h(\mathrm{x}, t)$. The instantaneous mean head gradient is then constant over the entire aquifer domain. This is accurate for cases in which flow unsteadiness is driven by seasonal changes in the mean head gradient. In that case the scale characterizing the variation of $\nabla\langle H\rangle$ is much larger than the plume size. This assumptions was adopted in other models [Naff et al., 1988, 1989; Rehfeldt and Gelhar, 1992] as well.

3. The flow and Darcy equations are solved by adopting a first-order approximation in $\sigma_{Y}^{2}$. The resulting mean velocity is given by [Dagan, 1982]

$$
\mathrm{U}(t)=\frac{K_{G}}{n} \mathrm{~J}(t),
$$

where $K_{G}=\exp [\langle Y\rangle]$ is the conductivity geometric mean and $n$ is the formation porosity.

Under assumptions (1) and (2) the velocity covariance function is obtained in the following form [Dagan et al., 1995]:

$u_{\imath}\left(\mathbf{x}, \mathbf{y}, t, t^{\prime}\right)=U_{\alpha}(t) U_{\beta}\left(t^{\prime}\right) \mathbf{v}_{y, \alpha \beta}(\mathbf{r}) \quad(\mathbf{r}=\mathbf{x}-\mathbf{y})$

where $U_{\alpha}(t), \alpha=1, \cdots, m$ is the $\alpha$ th component of the mean velocity field, $m$ is the space dimensionality, and $v_{i j, \alpha \beta}$ is the velocity tensor defined as the covariance between the velocity fluctuations $u_{i}(\mathbf{x})$ and $u_{j}(\mathbf{y})$, associated with a mean flow of velocity $\mathbf{U}$. The derivation of $v_{i j, \alpha \beta}$ is given in the appendix. The unsteadiness of the flow manifests itself through the time dependence of the mean velocity field. In the particular case of steady flow in the $x_{1}$ direction, i.e., $U_{\alpha}=U \delta_{1 \alpha}$, explicit 
expressions of the velocity covariance tensor, $U_{\imath}$, have been obtained by Rubin [1990] for a two-dimensional flow and isotropic exponential $C_{Y}$, by Rubin and Dagan [1992] for a threedimensional flow and an anisotropic exponential $C_{Y}$, and by Russo [1995] for a three-dimensional partially saturated flow and anisotropic exponential $C_{Y}$. Owing to symmetries present in equation (A1), explicit expressions of $v_{\iota \jmath, \alpha \beta}$ can be obtained in terms of $U_{t j}$ (see the appendix). Note that in order to distinguish between unsteady and steady state velocity covariance functions, we denote the last by capital letters, i.e., $U_{i j}$ is the steady state velocity covariance tensor while $u_{i j}$ is the tensor for the unsteady case.

Assuming ergodicity of the plume, i.e., that the plume is very large in the direction orthogonal to the long-term average mean flow direction [Dagan, 1991], the first-order expression for the trajectory of the plume centroid is given by [Dagan, 1984]

$$
\left\langle X_{j}(t)\right\rangle=\int_{0}^{t} U_{j}(\tau) d \tau=\frac{K_{G}}{n} \int_{0}^{t} J_{j}(\tau) d \tau
$$

where $J_{j}, j=1, \cdots, m$ are the components of the mean head gradient.

The spatial moments of the solute plume were obtained by the Lagrangian approach similar to that employed by Dagan [1984]:

$X_{t j}(t)=\left\langle X_{t}^{\prime}(t) X_{,}^{\prime}(t)\right\rangle=\int_{0}^{t} \int_{0}^{t} u_{v j}\left[\mathbf{X}\left(t^{\prime}\right), \mathbf{X}\left(t^{\prime \prime}\right), t^{\prime}, t^{\prime \prime}\right] d t^{\prime} d t^{\prime \prime}$

After replacing $\mathbf{X}$ in (5) by its expected value $\langle\mathbf{X}\rangle$, which is consistent with the order $O\left(\sigma_{Y}^{2}\right)$ of the sought solution, we obtain the following expression for $X_{i j}$ [Dagan et al., 1995]:

$$
X_{\imath,}(t)=\int_{0}^{t} \int_{0}^{t} U_{\alpha}\left(t^{\prime}\right) U_{\beta}\left(t^{\prime \prime}\right) v_{l, \alpha \beta}\left[\left\langle\mathbf{X}\left(t^{\prime}\right)\right\rangle-\left\langle\mathbf{X}\left(t^{\prime \prime}\right)\right\rangle\right] d t^{\prime} d t^{\prime \prime}
$$

Equation (6) encapsulates the effects of mean velocities not aligned with the long-term mean flow direction. For steady state, $U_{\alpha}$ is nonzero only for $\alpha=1$, and (6) reduces to the expression obtained previously by Dagan [1984].

Since this paper focuses on the application of the method to Borden, we specialize here the DBR model to axisymmetric formations, i.e., $I_{1}=I_{2}=I_{Y h} ; I_{3}=I_{Y v}$. The mean velocity field is assumed to oscillate in the horizontal plane with the vertical component $U_{3}=0$. The longitudinal direction is defined by the long-term time average of the mean velocity:

$$
\bar{U}=\frac{1}{t^{\prime \prime}} \int_{0}^{t^{\prime \prime}} U(t) d t=\frac{K_{G}}{n t^{\prime \prime}} \int_{0}^{r^{n}} J(t) d t
$$

where we assume that the duration $t^{\prime \prime}$ is sufficiently large for $\bar{U}$ to represent a meaningful time average.

DBR concentrated on the spatial moments in isotropic formations ( $f=1$ ), with seasonal fluctuations characterized by a single harmonic variation of $U$ around the long-term mean as follows:

$$
U_{1}=\vec{U} ; \quad U_{2}=\frac{\beta \bar{U}}{\lambda} \cos \left(\frac{t \bar{U}}{\lambda I_{Y h}}\right) ; \quad U_{3}=0 .
$$

The resulting trajectory of the plume centroid is

$\left\langle X_{1}(t)\right\rangle=\bar{U} t ;\left\langle X_{2}(t)\right\rangle=\beta I_{Y h} \sin \left(\frac{t \bar{U}}{\lambda I_{Y h}}\right) ; \quad\left\langle X_{3}\right\rangle=0$

The unsteadiness in this case is fully characterized by the parameters $\beta$, the dimensionless amplitude of the mean trajectory, and $\lambda=T \bar{U} /\left(2 \pi I_{Y h}\right)$, where $T$ is the period, respectively. The above study concluded that $\lambda$ and $\beta$ have a large impact on transverse and only a minor one on the longitudinal spread. The transverse dispersion coefficient $D_{22}=$ $(1 / 2) d X_{22} / d t$ and the associated macrodispersivity $A_{22}=$ $D_{22} / \bar{U}$ increase with time for $\beta>0$, reaching a constant nonzero Fickian limit at large time.

The essence of the DBR model is in the interaction between heterogeneity and unsteadiness. The asymptotic growth of the transverse spatial moment $X_{22}$ results from a simple physical mechanism: As the plume moves laterally it spreads in that direction at a rate determined by the steady longitudinal macrodispersion, which is much larger than the transverse one. A similar effect was investigated by Goode and Konikow [1990] for the interaction between unsteadiness of the mean flow and pore-scale dispersion. At any rate, the rate of spreading is proportional to the $\log$ conductivity variance $\sigma_{Y}^{2}$, and it also grows with the amplitude $\beta$.

This mechanism is different in principle from the one adopted by Rehfeldt and Gelhar [1992]. In their model the effects of unsteadiness and heterogeneity are additive, and the enhanced transverse spreading occurs in a homogeneous formation $\left(\sigma_{Y}^{2}=0\right)$ as well. This happens because they regard the unsteady motion as random, and their method assumes implicitly that concentration measurements are obtained by averaging over time. In our analysis of their method [see Dagan et al., 1995], we have emphasized that in field experiments such as at Borden, the major time variations have a seasonal nature and are characterized by a large timescale, while plume concentrations were measured over a short time, providing "instantaneous" snapshots. Under these conditions, unsteadiness in a homogeneous medium (with neglect of pore-scale dispersion) manifests itself in a translational motion of the plume with no spreading. Furthermore, the head gradient time variations were measured in detail, and they can be regarded as deterministic. Indeed, when the deterministic approach of Naff et al. [1988; 1989] was used by Farell et al. [1994], the resulting transverse spread was extremely small. For these reasons we concentrate subsequently on applying the DBR model solely.

\section{Discussion of Field Measurements at Borden Site and Summary of Data}

The application of the DBR model and comparison with field experiments requires identifying the following data: formation properties $\left(K_{G}, n, \sigma_{Y}^{2}, I_{Y h}, I_{Y v}\right)$, measured first and second spatial moments of the plume, $\mathbf{R}(t)$ and $S_{t j}(t)$, and unsteady mean gradient $\mathrm{J}(t)$ record. Herein is a brief discussion of the identification of these parameters.

\section{Formation Properties}

The Borden Site tracer experiments have been preceded by a sampling program in which a large number of hydraulic conductivity data were collected. The measurements were based on core extraction along two transects: The first, including 20 cores, roughly parallels the plume's trajectory (section 


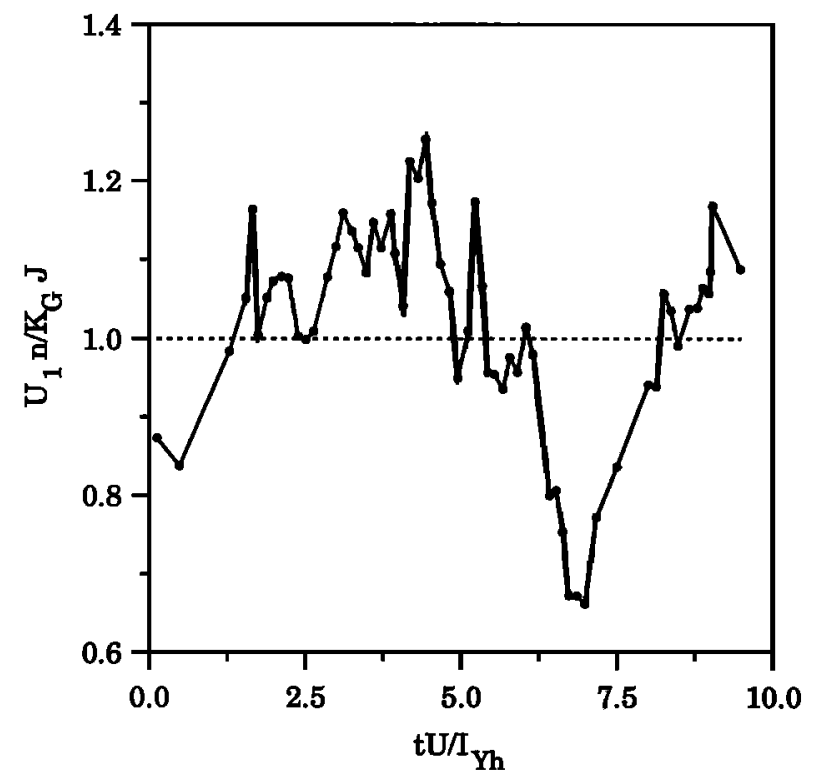

(a)

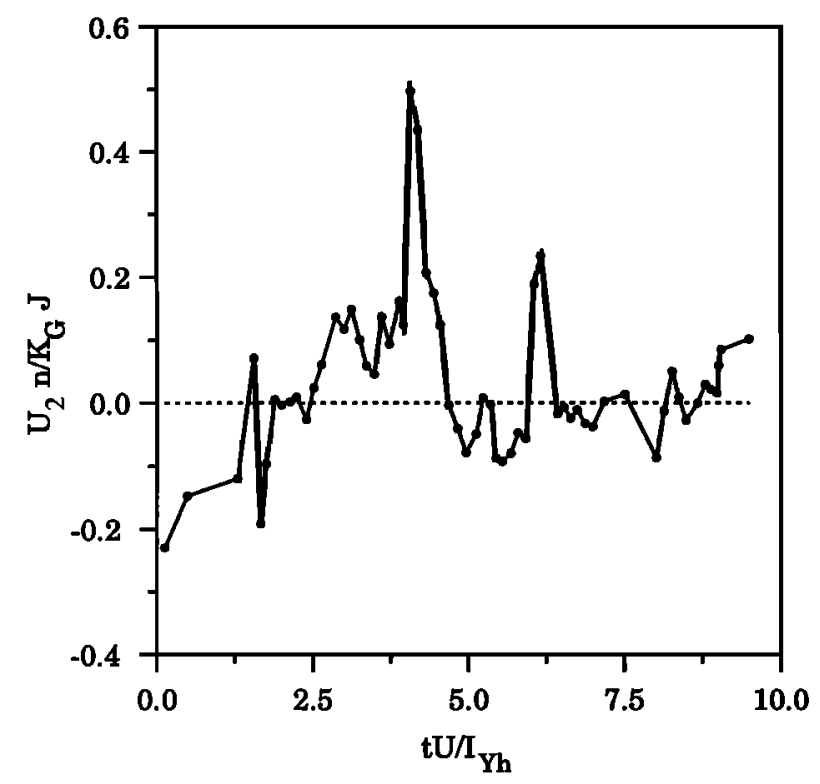

(b)

Figure 1. Dimensionless mean velocity as function of time, based on Figure 7a of Farrell et al. [1994]: (a) longitudinal component and (b) transverse component.

$A-A^{\prime}$ ), and the second, including 13 cores, is normal to the previous one (section B-B'). Hydraulic conductivities were measured in the laboratory using subsamples $0.05 \mathrm{~m}$ long taken from the cores and packed in the permeameter.

The first statistical analysis of the database was presented by Sudicky [1986], who concluded that the hydraulic conductivity is lognormally distributed. Recently, Woodbury and Sudicky [1991] revisited the original analysis, and the revised parameter estimate are given in Table 1. Woodbury and Sudicky adopted an exponential covariance model of the type given by (1) for the $\log$ conductivity with $I_{1}=I_{2}=I_{Y h}$ and $I_{3}=I_{Y v} \ll I_{Y h}$, with the parameter values corresponding to cross section $\mathbf{A}-\mathbf{A}^{\prime}$, which we adopt here as well. We note from Table 1 that the integral scales are characterized by large standard deviations.
The influence of this estimation uncertainty on transport modeling will be considered subsequently.

\section{Spatial Moments Analysis}

The common approach to computing the spatial moments, starting from irregular sampling of concentration, is to interpolate the measured data onto a regular grid followed by numerical quadrature [Aris, 1956]. The first analysis of BS1 data was performed by Freyberg [1986] using trapezoidal integration of solute concentrations measured along vertical boreholes, followed by projection of the values onto a regular horizontal grid and a numerical two-dimensional fourth-order quadrature scheme. Rajaram and Gelhar [1988] noted that the second-order moments computed by Freyberg are biased, as a result of incomplete plume sampling. In an attempt to remove this effect, some interpolation models have been proposed by several authors [Barry and Sposito, 1990; Rajaram and Gelhar, 1991; Thierrin and Kitanidis, 1994]. All authors agree that incomplete plume sampling and smoothing due to interpolation have a negligible impact on the second longitudinal plume moment $S_{11}$, but on the other hand, they have an appreciable impact on the transversal second moment $S_{22}$. The spatial moments of the Borden Site plume are close to the ergodic behavior due to the large number of vertical integral scales encompassed by the plume. This is also reflected by the uniform longitudinal motion of the centroid (see Figure 2a). Hence the DBR model is a suitable tool for representing the behavior of the Borden plume. In other situations, where the plume size is such that nonergodic behaviors are plausible, the analysis will have to be extended to incorporate such effects.

\section{Mean Head Gradient}

Freyberg [1986] pointed out that during the tracer gradient experiment, the mean head gradient experienced wide fluctuations. Unfortunately, the head gradients were not recorded regularly in BS1. In view of the data needs of our model, we present here two different ways for estimating the mean head gradient. The first method (I) consists of borrowing the head gradient data computed by Farrell et al. [1994] based on measurements collected during BS2. In this analysis the mean velocity field was taken parallel to the long-term mean head gradient. In agreement with previous observations [Freyberg, 1986], the horizontal mean velocity field is assumed aligned with the horizontal trajectory of the plume center of mass. This suggests that the horizontal anisotropy of the hydraulic conductivity is negligible with respect to the vertical anisotropy. The components of the mean velocity field obtained using the recorded head gradient time series are shown in Figures 1a and 1b. In agreement with experimental findings [Freyberg, 1986; Barry and Sposito, 1990; Rajaram and Gelhar, 1991], we assume $U_{3}=0$. Substituting the head gradient time series in (4) and performing a numerical quadrature, we obtain the trajectory of the plume center of mass shown by a solid line in Figure 2. It is seen from Figure 2b that the BS2 head gradient series does not lead to an accurate reproduction of the trajectory of the plume's centroid during the second and third years of BS1 survey of $R_{2}(t)$. This is probably caused by different climatic conditions during the two experiments.

The second method (II) consists of using the mean trajectory given by regression of (9) on the trajectory of the plume's centroid as computed by Freyberg [1986], which is shown in Figure 2. Using this method, we retain only the principal seasonal oscillation of the mean head gradient, while short-period 
oscillations are filtered out. The regression curve is shown in Figures $2 \mathrm{a}$ and $2 \mathrm{~b}$. The parameters found through this regression are $\beta=0.23$ and $\lambda=1.25$. On the basis of $I_{Y h}=5.14 \mathrm{~m}$ and $\bar{U}=0.09 \mathrm{~m} / \mathrm{day}$, these parameters lead to oscillation amplitude and period equal to $1.2 \mathrm{~m}$ and 71.66 days, respectively.

\section{Application to Anisotropic Formations: \\ The Borden Site Case}

\section{General}

In order to apply the DBR approach to Borden, anisotropy of the $\log$ conductivity covariance must be considered. This section expands the previous DBR model to anisotropic formations. The modification requires using the steady state ve-

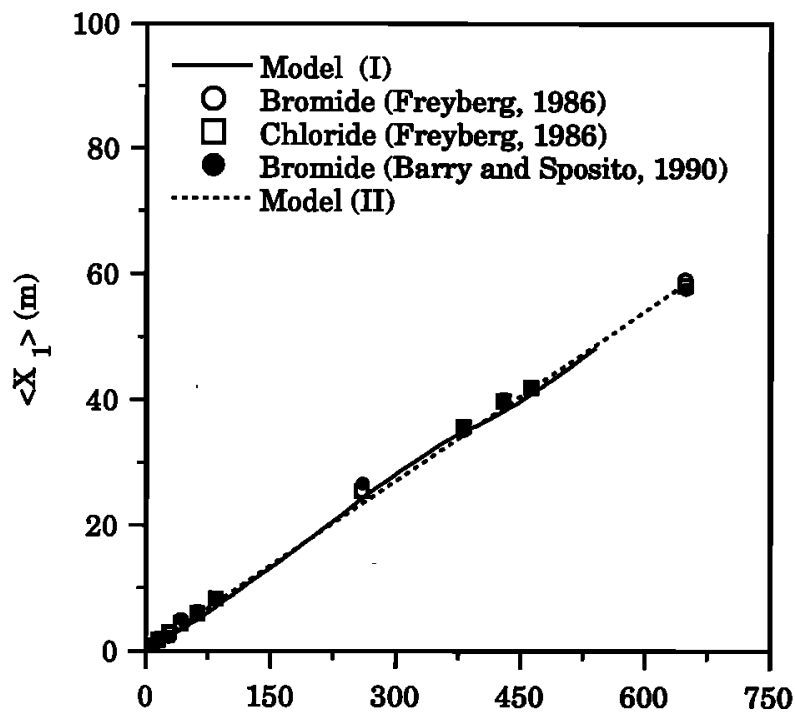

(a)

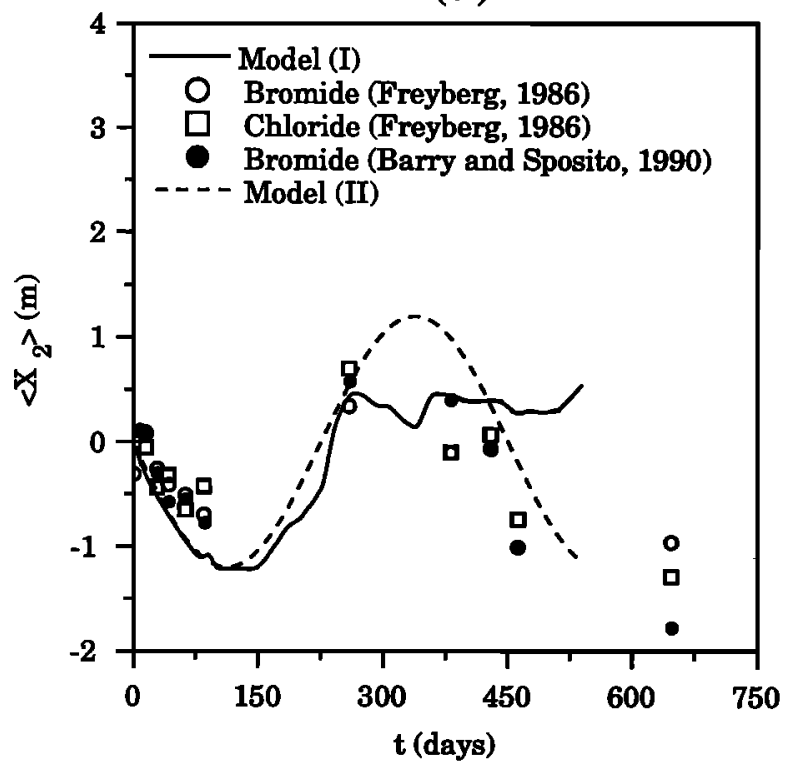

(b)

Figure 2. Horizontal components of the plume centroid coordinates versus time based on concentration measurements (symbols) as well as of models I (continuous lines) and II (dashed lines) for (a) horizontal longitudinal component and (b) horizontal transverse component.

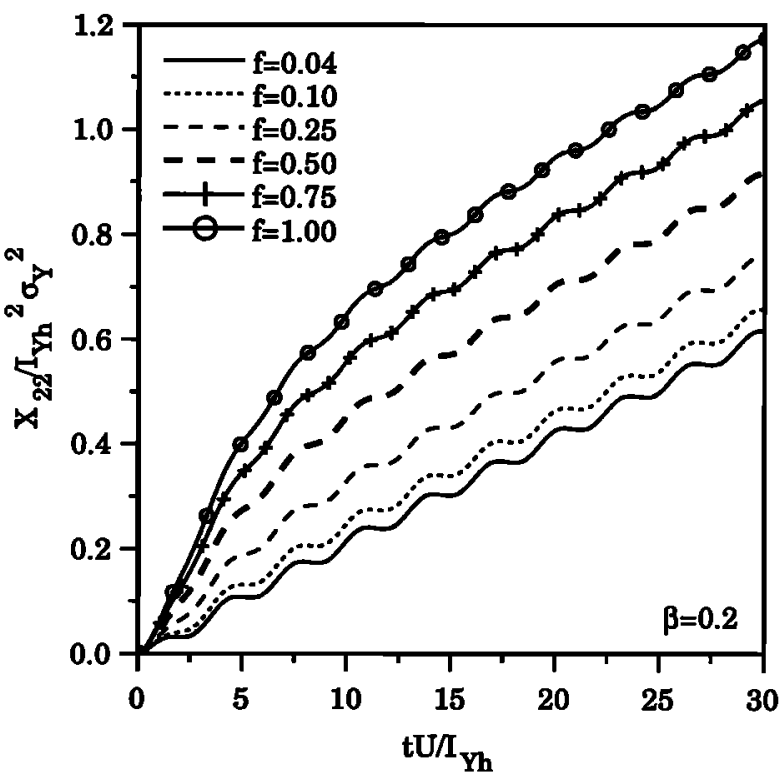

(a)

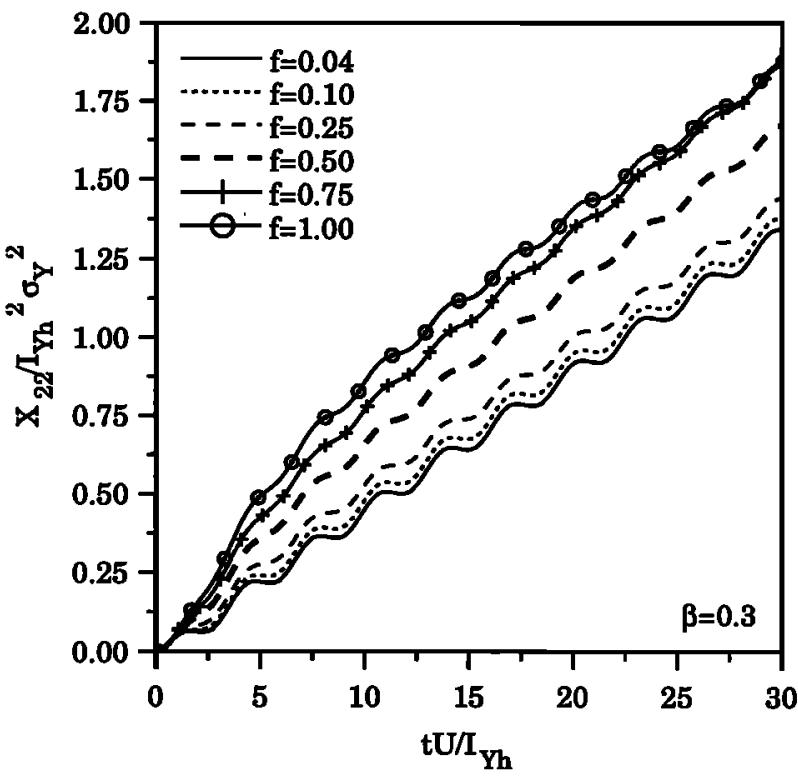

(b)

Figure 3. $X_{22}$ as a function of time (equation (6)) for a harmonic variation of the mean head gradient and for different degrees of anisotropy $f$ : (a) $\beta=0.2$ and (b) $\beta=0.3$. In all cases, $\lambda=1$.

locity covariances, which are reported by Dagan et al. [1995] and Rubin and Dagan [1992].

Figures $3 \mathrm{a}$ and $3 \mathrm{~b}$ show $X_{22}$ as a function of time for the single harmonic mean velocity field given by (8), for $\beta=0.2$ and $\beta=0.3$. In both cases, $\lambda=1$, while $f=I_{Y_{\nu}} / I_{Y h}$ varies between $f=0.04$, the value reported for Borden [Woodbury and Sudicky, 1991], and $f=1$, the isotropic case. Similar to steady state, $X_{22}$ increases with $f$, and the amplitude $\beta$ has a large impact on $X_{22}$. Transverse dispersivity reaches a Fickian asymptotic limit which depends on both $f$ and $\beta$. Figure 4 shows the asymptotic Fickian limit for transverse dispersivity. It can be seen that besides small fluctuations, probably due to 


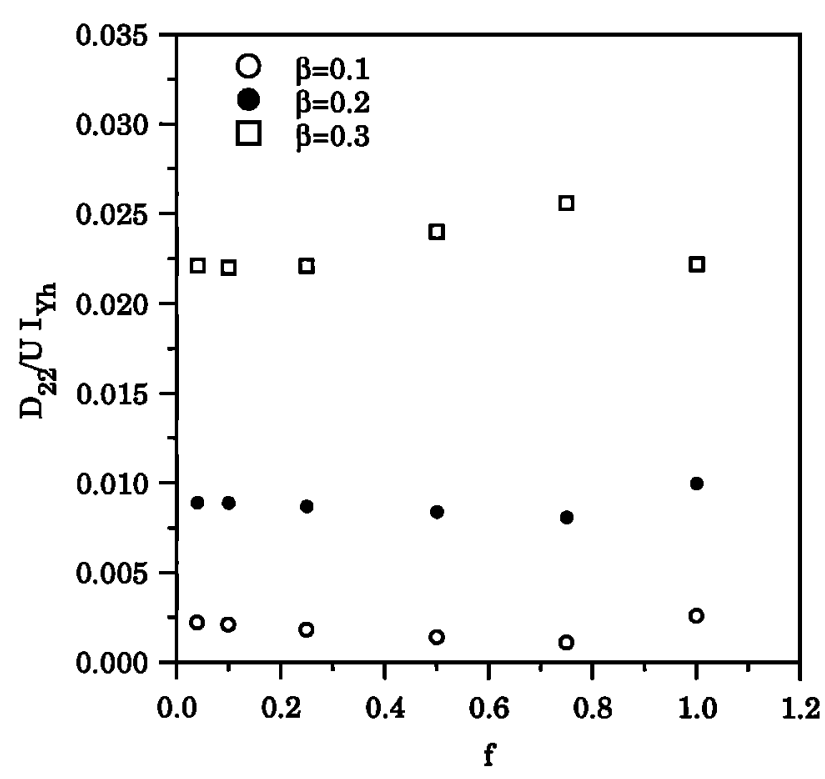

Figure 4. Fickian asymptotic limit for the transverse coefficient of dispersion $D_{22}$ as a function of $f$ for different mean velocity oscillation amplitude.

small numerical errors, the transverse asymptotic plume dispersivity is independent of $f$ and it increases rapidly with $\beta$, i.e., flow unsteadiness has a major impact on asymptotic transverse macrodispersivity, while the formation anisotropy plays a significant role only during the preasymptotic regime. An analysis of $X_{22}$ (see equation (6) and the appendix) reveals that for $\beta$ $=0.3$ and $f=0.04$, the major contribution is coming from $U_{11}^{*}\left(r_{1}, r_{2}, r_{3}\right)=U_{11}\left(r_{2}, r_{1}, r_{3}\right)$, i.e., the spreading mechanism which in steady state contributes only to $X_{11}$ (see Figures $5 \mathrm{a}$ and $5 \mathrm{~b}$ ). The contribution from $U_{12}^{*}$ is negligible, while that from $U_{22}$ (the steady state lateral mixing mechanism) is close to the one existing in steady state, which is negligible.

As $f$ increases, the contribution from $U_{22}$ increases while that from $U_{11}^{*}$ decreases. For $f=1$ the contribution from $U_{22}$ is larger than that from $U_{11}^{*}$ at earlier times, while the opposite happens at large times. This is in agreement with the fact that as $f$ increases, $U_{22}$ increases near the origin and decreases at large lags, while the opposite is true for $U_{11}^{*}$ [see Rubin and Dagan, 1992, Figures 3 and 4]. Although contributions from $U_{i j}, i, j=1,2$ change with $f$, the Fickian asymptotic limit of the transverse dispersion coefficient $D_{22}$ is quite constant inside the range $0<f \leq 1$ (Figure 4).

\section{The Borden Site Case: Discussion and Results}

The previous results assume a single harmonic head gradient oscillation. Such a situation is seldom observed in nature, where short-period oscillations are superimposed on seasonal variations that in turn may change through the years. A necessary condition for reaching a Fickian limit characterized by a constant macrodispersivity is for the periodicities to be stationary. In natural conditions, successive years may show different behavior of the gradient oscillations, which prevents transport from converging to well-defined Fickian limits. The dispersivity can change through the years, depending on head gradient oscillations, and hence the computation of asymptotic dispersivities based on few years of survey may be difficult and in some cases intractable. This is illustrated by Figures $6 \mathrm{a}$ and $6 \mathrm{~b}$, which display $X_{22}(t)$ for the actual record of $\mathbf{J}$ based on method I, for method II, as well as the inferred experimental $S_{22}$ values reported by different authors. It is seen that for method I, a quick increase of the transverse dispersivity occurs at $t \simeq 230$ days, which is followed by smaller transverse dispersivity induced by smoother gradient oscillations (see Figure 6a).

Since the impact of transients on macrodispersion depends on the oscillations of the trajectory of the plume centroid, and also in order to assess the impact of the different conditions during the BS1 and BS2 tests, we applied the DBR model using both models I and II.

The estimates of formation parameters we employed in our model are those suggested by Woodbury and Sudicky [1991] (see Table 1). Note from (A1) that the nugget effect does not

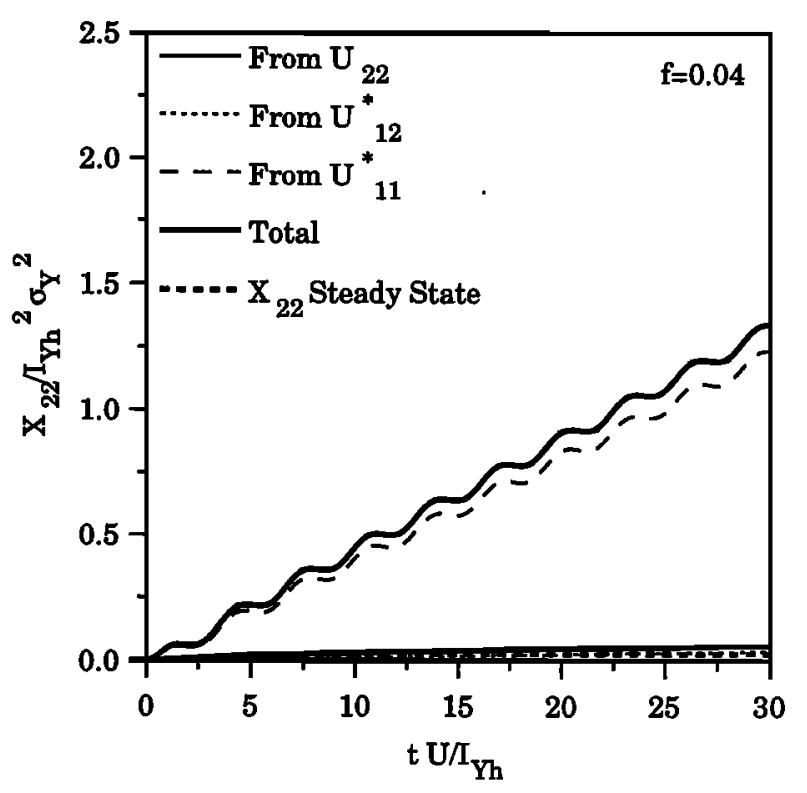

(a)

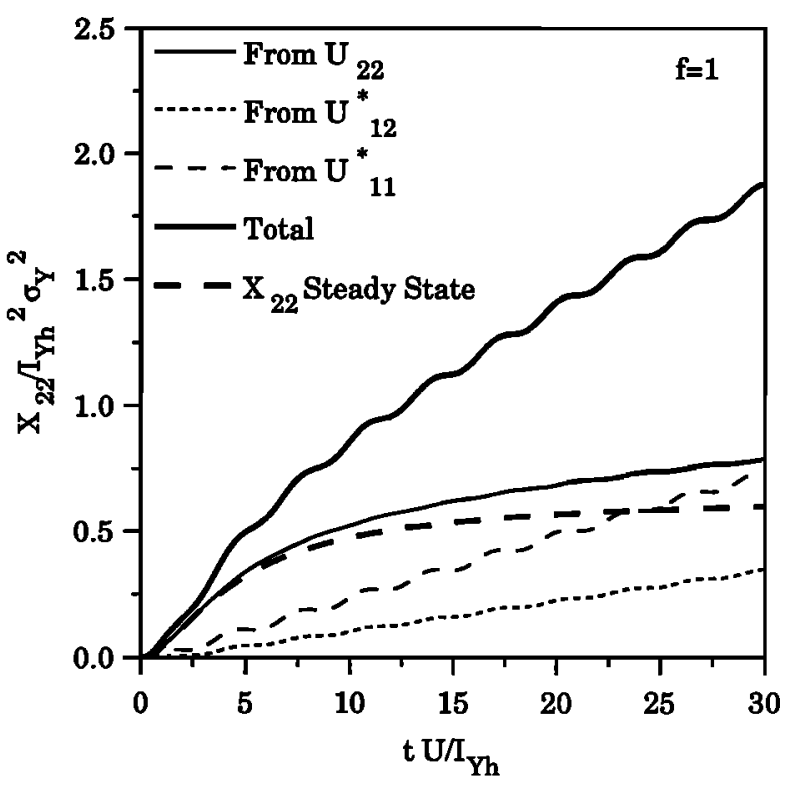

(b)

Figure 5. $X_{22}$ versus time for (a) $f=0.04$ and (b) $f=1$. Contributions from $U_{i j}, i, j=1,2$ are shown. 
contribute to spreading, hence the value for $\sigma_{Y}^{2}$ is obtained by subtracting the "nugget" $\sigma_{0}^{2}$ from the total variance $\hat{\sigma}_{Y}^{2}$ (Table 1).

Model parameters are affected by measurement errors, which add uncertainty to the computation of plume moments. Woodbury and Sudicky [1991] computed the mean and standard deviation of the formation parameters $I_{Y h}, I_{Y v}$, and $\sigma_{Y}^{2}$ (Table 1). The range of variations of $I_{Y h}$ and $I_{Y v}$ within their 95\% intervals of confidence suggest that $f$ can actually vary between 0.014 and 0.11 , which, according to Figure 3, has a negligible impact on $X_{22}$. Hence we shall adopt for $f$ its ex-

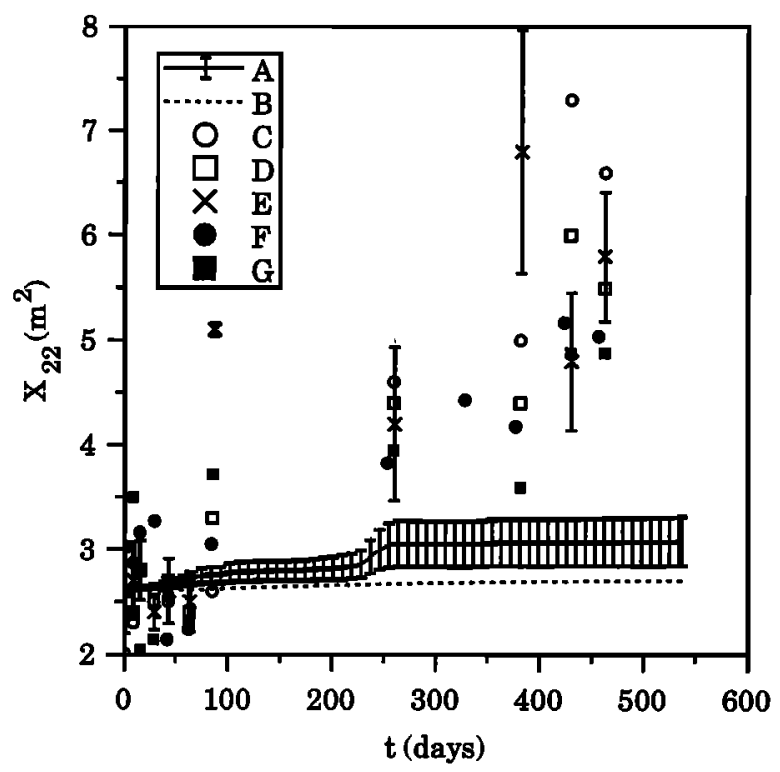

(a)

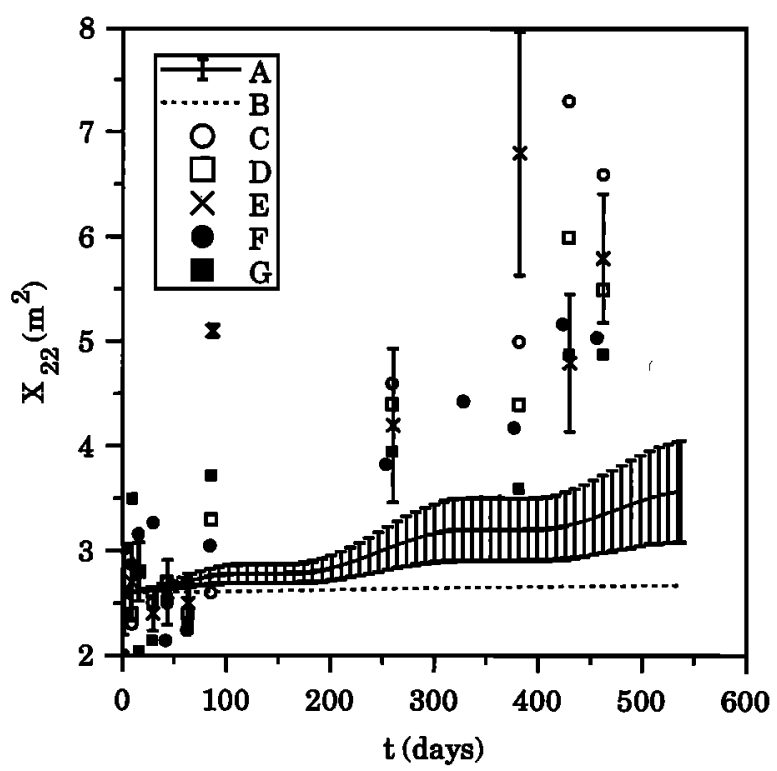

(b)

Figure 6. $E\left[X_{22}\right]$ and its $95 \%$ interval of confidence as function of time for (a) model I and (b) model II. In all cases, B denotes steady state flow $\left(f=0.04, I_{Y h}=5.14 \mathrm{~m}\right) ; \mathrm{C}$, bromide [Freyberg, 1986]; D, chloride [Freyberg, 1986]; E, bromide with $95 \%$ interval of confidence [Barry and Sposito, 1990]; F, bromide [Rajaram and Gelhar, 1991]; and G, bromide [Thierrin and Kitanidis, 1994].
Table 2. Expected Values $(E)$ and Standard Deviation (s.d.) of the Model Parameters Used for the Simulations

\begin{tabular}{ll}
\hline \multicolumn{1}{c}{ Parameter } & Value \\
\hline$E[f]$ & 0.04 \\
$E\left[I_{Y h}\right], \mathrm{m}$ & 5.14 \\
s.d. $\left[I_{Y h}\right], \mathrm{m}$ & 1.17 \\
$E\left[\sigma_{Y}^{2}\right]$ & 0.172 \\
s.d. $\left[\sigma_{Y}^{2}\right]$ & 0.0225 \\
$E[n]$ & 0.33 \\
$E[\bar{U}], \mathrm{m} / \mathrm{day}$ & 0.09 \\
s.d. $[\bar{U}], \mathrm{m} / \mathrm{day}$ & 0.009 \\
\hline
\end{tabular}

pected value $E[f]=0.04$ (see Table 2). Another source of uncertainty is the long-term time average of the mean velocity that can be estimated directly from the recorded trajectory of the plume centroid. Substituting in (7) the value of $\bar{J}$ computed by Farrell et al. [1994] and the expected values of $K_{G}$ and $n$ computed by Sudicky [1986] and Woodbury and Sudicky [1991], which are shown in Table 2 , we obtain $\bar{U}=0.09043 \mathrm{~m} /$ day. Since this value compares favorably with the value of 0.09 $\mathrm{m} /$ day computed by several authors [Freyberg, 1986; Barry and Sposito, 1990; Rajaram and Gelhar, 1991] from moments analysis, we adopt it as the estimate of $\bar{U}$. As for the variance of $\bar{U}$, we have used the values of the standard deviations of $R_{1}(t)$, the centroid trajectory in the mean flow direction, as given by Barry and Sposito [1990]. A simplified analysis of their data leads to an average standard deviation value $S D(\bar{U})=0.09$, which is also given in Table 2. To assess the impact of parameter estimation errors upon prediction of $X_{i}$ by the theoretical model, we have recast (6) in the following way:

$$
X_{y}(t)=\sigma_{Y}^{2} I_{Y h}^{2} X_{i j}^{\prime}\left(t^{\prime}\right)
$$

where $X_{i j}^{\prime}$ is the dimensionless second-order centered plume moment and $t^{\prime}=t \bar{U} / I_{Y h}$ is the dimensionless time. The expected values of the plume moments are computed by

$$
E\left[X_{t}(t)\right]=\iiint \sigma_{Y}^{2} I_{Y h}^{2} X_{t j}^{\prime}\left(t^{\prime}\right) p\left(I_{Y h}, \bar{U}, \sigma_{Y}^{2}\right) d I_{Y h} d \bar{U} d \sigma_{Y}^{2}
$$

where $p$ is the joint probability distribution function of the parameter estimates. For sake of illustration we shall assume the parameters to be statistically independent and normally distributed such that $p$ is given by the product of the marginals $p\left(I_{Y h}, \bar{U}, \sigma_{Y}^{2}\right)=p_{I_{Y h}}\left(I_{Y h}\right) p_{\bar{U}}(\bar{U}) p \sigma_{Y}^{2}\left(\sigma_{Y}^{2}\right)$. Since $X_{i j}$ depends linearly on $\sigma_{Y}^{2}$, equation (11) reduces to

$$
E\left[X_{y}(t)\right]=E\left[\sigma_{Y}^{2}\right] \iint I_{Y h}^{2} X_{y j}^{\prime}\left(t^{\prime}\right) p_{l y h}\left(I_{y h}\right) p_{\bar{U}}(\bar{U}) d I_{Y h} d \bar{U}
$$

and the variance of $X_{\iota j}$ is given by

$$
\begin{aligned}
& \operatorname{Var}\left[X_{l j}\right]=\left\{\operatorname{Var}\left[\sigma_{Y}^{2}\right]+\left[E\left(\sigma_{Y}^{2}\right)\right]^{2}\right\} \\
& \cdot \iint I_{Y h}^{4} X_{i j}^{\prime 2}\left(t^{\prime}\right) p_{I_{Y h}}\left(I_{Y h}\right) p_{\bar{U}}(\bar{U}) d I_{Y h} d \bar{U}-E\left[X_{i j}\right]^{2}
\end{aligned}
$$

where $\operatorname{Var}\left[\sigma_{Y}^{2}\right]$ is the estimation variance of $\sigma_{Y}^{2}$. Owing to the nonlinear dependence of $X_{i j}^{\prime}$ on $\bar{U}$ and $I_{Y h}$, equations (12) and (13) were computed by numerical quadrature. Computation of 
$E\left[X_{u j}\right]$ and their $95 \%$ interval of confidence is performed with models I and II.

Figure 6a shows $E\left[X_{22}\right]$ and its interval of confidence for model $\mathrm{I}$, while those corresponding to model II are given in Figure $6 \mathrm{~b}$. The jump in $X_{22}$ at $t \simeq 230$ days results from the quick rotation of the mean head gradient toward the direction $x_{2}$. At $t>250$ days, $X_{22}$ grows at a lower rate due to the gradient rotation back toward the direction $x_{1}$ and hence the elimination of the enhanced lateral growth mechanism. At $t>$ 250 days the trajectory of the plume centroid shows small deviations from the $x_{1}$ direction and $X_{22}$ resumes its previous growth rate.

It is seen that accounting for the transient behavior of the head gradient leads to $X_{22}$ values much larger than those based on the steady state model. Nevertheless, despite the large scatter of $S_{22}$ estimates, it is seen that the theoretical transient model still underpredicts $S_{22}$ at large travel times.

\section{Summary and Conclusions}

The conclusions of this paper are as follows:

1. We provided a methodology to assess the impact of flow unsteadiniess on transport of solute in heterogeneous porous formations based on head gradient fluctuations in time. The method provides the preasymptotic behavior of transverse macrodispersivity, which is particularly important for transport under natural conditions.

2. We applied the method to the 1982-1985 Borden Site tracer experiment (BS1) using a three-dimensional anisotropic $\log$ conductivity field.

3. Flow unsteadiness of the type analyzed here has a minor impact on the longitudinal plume second-order moment. Differences less than $5 \%$ between theoretical steady state and unsteady solutions were found for times larger than 100 days. Consequently, we concentrated on the transverse spreading in the horizontal plane solely.

4. The impact of flow unsteadiness on transverse mixing is more significant, and it depends on formation anisotropy and on the magnitude and period of the head gradient oscillations.

5. For the Borden Site the estimates of $S_{22}$ by various authors display a large scatter. Nevertheless, the theoretical $X_{22}$ underpredicts the experimental data. Thus we feel that the large transverse spreading in this field experiment could not be completely explained by the observed unsteadiness of the head gradient.

6. Since the transverse spreading is sensitive to variations in phase and amplitude of the head gradient oscillation, it is recommended to record accurately the head gradient time series in future field experiments.

\section{Appendix}

The velocity tensor employed in (3) is given by

$v_{l, \alpha \beta}(\mathbf{r})=\delta_{l \alpha} \delta_{j \beta} C_{Y}(\mathbf{r})+\delta_{j \beta} \frac{\partial^{2} P}{\partial r_{\imath} \partial r_{\alpha}}+\delta_{l \alpha} \frac{\partial^{2} P}{\partial r_{j} \partial r_{\beta}}-\frac{\partial^{4} Q}{\partial r_{l} \partial r_{\alpha} \partial r_{j} \partial r_{\beta}}$

where $\delta$ is the Kronecker function and $P$ and $Q$ are two functions satisfying the following differential equations [Dagan, 1989; Rubin, 1990]: $\nabla^{2} P=-C_{Y} ; P(0)=0$; and $\nabla^{2} Q=P$; $Q(0)=0$.

The horizontal components of the tensor $u_{i j}$ (equation (3)) assumes for the three dimensional anisotropic case the following form:

$$
\begin{aligned}
& u_{11}\left(\mathbf{r}, t^{\prime}, t^{\prime \prime}\right)=U_{1}\left(t^{\prime}\right) U_{1}\left(t^{\prime \prime}\right)\left\{C_{Y}(\mathbf{r})+2 P_{11}(\mathbf{r})-Q_{1111}(\mathbf{r})\right\} \\
& +\left[U_{1}\left(t^{\prime}\right) U_{2}\left(t^{\prime \prime}\right)+U_{1}\left(t^{\prime \prime}\right) U_{2}\left(t^{\prime}\right)\right]\left\{P_{12}(\mathbf{r})-Q_{1112}(\mathbf{r})\right\} \\
& +\left[U_{1}\left(t^{\prime}\right) U_{3}\left(t^{\prime \prime}\right)+U_{1}\left(t^{\prime \prime}\right) U_{3}\left(t^{\prime}\right)\right] \cdot\left\{P_{13}(\mathbf{r})-Q_{1113}(\mathbf{r})\right\} \\
& +U_{2}\left(t^{\prime}\right) U_{2}\left(t^{\prime \prime}\right)\left\{-Q_{1122}(\mathbf{r})\right\} \\
& +\left[U_{2}\left(t^{\prime}\right) U_{3}\left(t^{\prime \prime}\right)+U_{2}\left(t^{\prime \prime}\right) U_{3}\left(t^{\prime}\right)\right]\left\{-Q_{1123}(\mathbf{r})\right\} \\
& +U_{3}\left(t^{\prime}\right) U_{3}\left(t^{\prime \prime}\right)\left\{-Q_{1133}(\mathbf{r})\right\} \\
& u_{12}\left(\mathbf{r}, t^{\prime}, t^{\prime \prime}\right)=U_{1}\left(t^{\prime}\right) U_{1}\left(t^{\prime \prime}\right)\left\{P_{12}(\mathbf{r})-Q_{1112}(\mathbf{r})\right\} \\
& +U_{1}\left(t^{\prime}\right) U_{2}\left(t^{\prime \prime}\right)\left\{C_{Y}(\mathbf{r})+P_{11}(\mathbf{r})+P_{22}(\mathbf{r})-Q_{1122}(\mathbf{r})\right\} \\
& +U_{1}\left(t^{\prime \prime}\right) U_{2}\left(t^{\prime}\right)\left\{-Q_{1122}(\mathbf{r})\right\}+U_{1}\left(t^{\prime}\right) U_{3}\left(t^{\prime \prime}\right) \\
& \cdot\left\{P_{23}(\mathbf{r})-Q_{1123}(\mathbf{r})\right\}+U_{1}\left(t^{\prime \prime}\right) U_{3}\left(t^{\prime}\right) \\
& \cdot\left\{-Q_{1123}(\mathbf{r})\right\}+U_{2}\left(t^{\prime}\right) U_{2}\left(t^{\prime \prime}\right)\left\{P_{12}(\mathbf{r})-Q_{1222}(\mathbf{r})\right\} \\
& +U_{2}\left(t^{\prime}\right) U_{3}\left(t^{\prime \prime}\right)\left\{P_{13}(\mathbf{r})-Q_{1223}(\mathbf{r})\right\}+U_{2}\left(t^{\prime \prime}\right) U_{3}\left(t^{\prime}\right) \\
& \cdot\left\{Q_{1223}(\mathbf{r})\right\}+U_{3}\left(t^{\prime}\right) U_{3}\left(t^{\prime \prime}\right)\left\{-Q_{1233}(\mathbf{r})\right\} \\
& u_{22}\left(\mathbf{r}, t^{\prime}, t^{\prime \prime}\right)=U_{1}\left(t^{\prime}\right) U_{1}\left(t^{\prime \prime}\right)\left\{-Q_{1122}(\mathbf{r})\right\} \\
& +\left[U_{1}\left(t^{\prime}\right) U_{2}\left(t^{\prime \prime}\right)+U_{1}\left(t^{\prime \prime}\right) U_{2}\left(t^{\prime}\right)\right]\left\{P_{12}(\mathbf{r})-Q_{1222}(\mathbf{r})\right\} \\
& +\left[U_{1}\left(t^{\prime}\right) U_{3}\left(t^{\prime \prime}\right)+U_{1}\left(t^{\prime \prime}\right) U_{3}\left(t^{\prime}\right)\right]\left\{-Q_{1223}(\mathbf{r})\right\} \\
& +U_{2}\left(t^{\prime}\right) U_{2}\left(t^{\prime \prime}\right)\left\{C_{\mathrm{r}}(\mathbf{r})+2 P_{22}(\mathbf{r})-Q_{2222}(\mathbf{r})\right\} \\
& +\left[U_{2}\left(t^{\prime}\right) U_{3}\left(t^{\prime \prime}\right)+U_{2}\left(t^{\prime \prime}\right) U_{3}\left(t^{\prime}\right)\right] \\
& \cdot\left\{P_{23}(\mathbf{r})-Q_{2233}(\mathbf{r})\right\}+U_{3}\left(t^{\prime}\right) U_{3}\left(t^{\prime \prime}\right)\left\{-Q_{2233}(\mathbf{r})\right\}
\end{aligned}
$$

Assuming the mean velocity field given by $\mathbf{U}(t)=\left(U_{1}(t)\right.$, $\left.U_{2}(t), 0\right)$ and considering the expressions for $U_{1 j}$ (i.e., the steady state velocity covariance function for $\mathbf{U}=(U, 0,0))$, given by Rubin and Dagan [1992], equations (A2), (A3), and (A4) simplify to

$$
\begin{aligned}
u_{11}\left(\mathbf{r}, t^{\prime}, t^{\prime \prime}\right)= & U_{1}\left(t^{\prime}\right) U_{1}\left(t^{\prime \prime}\right) U_{11}(\mathbf{r}) \\
& +\left[U_{1}\left(t^{\prime}\right) U_{2}\left(t^{\prime \prime}\right)+U_{1}\left(t^{\prime \prime}\right) U_{2}\left(t^{\prime}\right)\right] U_{12}(\mathbf{r}) \\
& +U_{2}\left(t^{\prime}\right) U_{2}\left(t^{\prime \prime}\right) U_{22}(\mathbf{r}) \\
u_{12}\left(\mathbf{r}, t^{\prime}, t^{\prime \prime}\right)= & U_{1}\left(t^{\prime}\right) U_{1}\left(t^{\prime \prime}\right) U_{12}(\mathbf{r})+U_{1}\left(t^{\prime}\right) U_{2}\left(t^{\prime \prime}\right) \\
& \cdot\left\{U_{22}(\mathbf{r})-P_{33}(\mathbf{r})\right\}+U_{1}\left(t^{\prime \prime}\right) U_{2}\left(t^{\prime}\right) U_{22}(\mathbf{r}) \\
& +U_{2}\left(t^{\prime}\right) U_{2}\left(t^{\prime \prime}\right) U_{12}^{*}(\mathbf{r}) \\
u_{22}\left(\mathbf{r}, t^{\prime}, t^{\prime \prime}\right)= & U_{1}\left(t^{\prime}\right) U_{1}\left(t^{\prime \prime}\right) U_{22}(\mathbf{r}) \\
& +\left[U_{1}\left(t^{\prime}\right) U_{2}\left(t^{\prime \prime}\right)+U_{1}\left(t^{\prime \prime}\right) U_{2}\left(t^{\prime}\right)\right] U_{12}^{*}(\mathbf{r}) \\
& +U_{2}\left(t^{\prime}\right) U_{2}\left(t^{\prime \prime}\right) U_{11}^{*}(\mathbf{r})
\end{aligned}
$$

where $U_{i j}^{*}\left(r_{1}, r_{2}, r_{3}\right)=U_{i j}\left(r_{2}, r_{1}, r_{3}\right)$ and $P_{33}$ assumes the following expression:

$$
P_{33}(\mathbf{r})=\sigma_{Y}^{2} \int_{0}^{\infty} \frac{2 f k \sqrt{1+k^{2}}-k^{2}\left(1+f^{2}\right)-1}{\sqrt{1+k^{2}}\left[1+\left(1-f^{2}\right) k^{2}\right]^{2}} \bar{J}_{0}(k \rho) k d k
$$

with $\rho=\sqrt{r_{1}^{2}+r_{2}^{2}}$ and $\bar{J}_{0}$ the Bessel function of order zero. 


\section{References}

Aris, R., On the dispersion of a solute in a fluid flowing through a tube, Proc. R. Soc. London A, 235, 67-78, 1956.

Barry, D. A., and G. Sposito, Three-dimensional statistical moment analysis of the Stanford/Waterloo Borden tracer data, Water Resour. Res., 26(8), 1735-1747, 1990.

Burr, D. T., E. A. Sudicky, and R. L. Naff, Nonreactive and reactive solute transport in three-dimensional heterogeneous porous media: Mean displacement, plume spreading, and uncertainty, Water Resour. Res., 30(3), 791-815, 1994.

Dagan, G., Stochastic modeling of groundwater flow by unconditional and conditional probabilities, 2, The solute transport, Water Resour. Res., 18(4), 835-848, 1982.

Dagan, G., Solute transport in heterogeneous porous formations, $J$. Fluid Mech., 145, 151-177, 1984.

Dagan, G., Time-dependent macrodispersion for solute transport in anisotropic heterogeneous aquifers, Water Resour. Res., 24(9), 1491$1500,1988$.

Dagan, G., Flow and Transport in Porous Formations, Springer-Verlag, New York, 1989.

Dagan, G., Dispersion of passive solute in non-ergodic transport by steady velocity fields in heterogeneous formations, J. Fluid Mech., 223, 197-210, 1991.

Dagan, G., Comment on "Nonreactive and reactive solute transport in three-dimensional heterogeneous porous media: Mean displacement, plume spreading, and uncertainty, by T. D. Burr, E. A. Sudicky, and R. L. Naff," Water Resour. Res., 31(5), 1439-1441, 1995.

Dagan, G., A. Bellin, and Y. Rubin, Lagrangian analysis of transport in heterogeneous formations under transient flow conditions, Water Resour. Res., 32(4), 891-899, 1996.

Farrell, D. A., A. D. Woodbury, E. A. Sudicky, and M. O. Rivett, Stochastic and deterministic analysis of dispersion in unsteady flow at the Borden tracer-test site, Ontario, Canada, J. Contam. Hydrol., 15, 159-185, 1994.

Fiori, A., Finite Peclet extensions of Dagan's solutions to transport in anisotropic heterogeneous formations, Water Resour. Res., 32(1), 193-198, 1996.

Freyberg, D. L., A natural gradient experiment on solute transport in a sand aquifer, 2, Spatial moments and the advection and dispersion of nonreactive tracers, Water Resour. Res., 22(13), 2031-2046, 1986.

Goode, D. J., and L. Konikow, Apparent dispersion in transient groundwater flow, Water Resour. Res., 26(10), 2339-2351, 1990.

Mackay, D. M., D. L. Freyberg, P. V. Roberts, and J. A. Cherry, A natural gradient experiment on solute transport in a sand aquifer, 1 , Approach and overview of plume movement, Water Resour. Res., 22(13), 2017-2029, 1986.

Naff, R. L., T. C. J. Yeh, and M. W. Kemblowski, A note on the recent natural gradient tracer test at the Borden site, Water Resour. Res., 24(12), 2099-2103, 1988.

Naff, R. L., T. C. Yeh, and M. W. Kemblowski, Reply to "Comment on "A note on the recent natural gradient tracer test at the borden site by R. L. Naff, T. C. Yeh, and M. W. Kemblowski,' by G. Dagan," Water Resour. Res., 25(12), 2523-2525, 1989.

Rajaram, H., and L. W. Gelhar, Field application of stochastic models of dispersive transport, Tech. Rep. CR-813359-01, edited by R. S. Kerr, Environ. Res. Lab., U.S. Environ. Prot. Agency, Washington, D. C., 1988.

Rajaram, H., and L. W. Gelhar, Three-dimensional spatial moments analysis of the Borden tracer test, Water Resour. Res., 27(6), 12391251, 1991.

Rehfeldt, K. R., and L. W. Gelhar, Stochastic analysis of dispersion in unsteady flow in heterogeneous aquifers, Water Resour. Res., 28(8), 2085-2099, 1992.

Rubin, Y., Stochastic modeling of macrodispersion in heterogeneous porous media, Water Resour. Res., 26(1), 133-141, 1990. (Correction, Water Resour. Res., 26(10), 2631, 1990.)

Rubin, Y., and G. Dagan, A note on head and velocity covariances in three-dimensional flow through heterogeneous anisotropic porous media, Water Resour. Res., 28(5), 1463-1470, 1992. (Correction, Water Resour. Res., 31(6), 1613, 1995.)

Russo, D., Stochastic analysis of the velocity covariance and the displacement covariance tensors in partially saturated heterogeneous anisotropic porous formations, Water Resour. Res., 31(7), 1647-1658, 1995.

Sudicky, E. A., A natural gradient experiment on solute transport in a sand aquifer: Spatial variability of hydraulic conductivity and its role in the dispersion process, Water Resour. Res., 22(13), 2069-2082, 1986.

Thierrin, J., and P. K. Kitanidis, Solute dilution at the Borden and Cape Cod groundwater tracer tests, Water Resour. Res., 30(11), 2883-2890, 1994.

Woodbury, A. D., and E. A. Sudicky, The geostatistical characteristics of the Borden aquifer, Water Resour. Res., 27(4), 533-546, 1991.

A. Bellin, Dipartimento di Ingegneria Civile ed Ambientale, Università di Trento, via Mesiano 77, I-38050 Trento, Italy. (e-mail: Alberto.Bellin@ing.unitn.it)

G. Dagan, Faculty of Engineering, Tel Aviv University, P. O. Box 39040, Tel Aviv 69978, Israel.

Y. Rubin, Department of Civil Engineering, University of California at Berkeley, Berkeley, CA 94720. (e-mail: rubin@ce.berkeley.edu)

(Received November 6, 1995; revised April 8, 1996; accepted May 24, 1996.) 